\title{
Embryonic Decellularized Cardiac Scaffold Supports Embryonic Stem Cell Differentiation to Produce Beating Cardiac Tissue
}

\author{
Cree Chamberland, ${ }^{1}$ Almudena Martinez-Fernandez, ${ }^{1,2}$ \\ Rosanna Beraldi, ${ }^{2,3}$ and Timothy J. Nelson ${ }^{2,3,4,5}$ \\ ${ }^{1}$ Division of Cardiovascular Diseases, Department of Medicine, Mayo Clinic, Rochester, MN 55905, USA \\ ${ }^{2}$ Center for Regenerative Medicine, Mayo Clinic, Rochester, MN 55905, USA \\ ${ }^{3}$ Division of General Internal Medicine, Mayo Clinic, Rochester, MN 55905, USA \\ ${ }^{4}$ Department of Molecular Pharmacology and Experimental Therapeutics, Mayo Clinic, Rochester, MN 55905, USA \\ ${ }^{5}$ Transplant Center, Mayo Clinic, Rochester, MN 55905, USA
}

Correspondence should be addressed to Timothy J. Nelson; nelson.timothy@mayo.edu

Received 12 November 2013; Accepted 4 December 2013; Published 31 March 2014

Academic Editors: R. Dressel and S. M. Hwang

Copyright (c) 2014 Cree Chamberland et al. This is an open access article distributed under the Creative Commons Attribution License, which permits unrestricted use, distribution, and reproduction in any medium, provided the original work is properly cited.

\begin{abstract}
Regenerative medicine offers a curative approach to treating heart disease through multiple emerging therapeutic concepts. Decellularized organ scaffolds are being optimized to guide and spatially organize stem cell differentiation in efforts to rebuild functional tissues. Additionally, pluripotent stem cells offer a transformative cell source to differentiate into the full spectrum of cellular building blocks. Adult cardiac tissues have been used as extracellular scaffolds as a proof of principle; however, matching the developmental stages of embryonic scaffold with primitive cardiac progenitors may be used to optimize the differentiation and maturation of bioengineered cardiac tissues. Our novel approach uses embryo-derived decellularized hearts as scaffolds to promote embryonic stem cell differentiation. Further, we determined that agitation with $0.25 \%$ sodium dodecyl sulfate (SDS) solution was the most effective protocol to maintain matrix integrity while eliminating endogenous cells. The scaffolds were successfully reseeded with different cellular sources derived from pluripotent stem cells to achieve beating cardiac tissues characterized by endothelial, cardiac, and smooth muscle markers. Therefore, embedding stem cells within a tissue-specific environment matched to the developmental stage of the progenitors may offer a practical solution for stem-cell-derived applications such as disease modeling, pharmaceutical safety testing, and screening of novel therapeutic targets.
\end{abstract}

\section{Introduction}

Regenerative medicine offers a new paradigm for the treatment of heart disease through replacement of damaged cardiac tissue with adult, embryonic, or induced pluripotent stem cells (iPSC). Due to a limited capacity of the heart to innately regenerate, multiple strategies are being explored and tested to optimize stem cell-based regeneration [1]. The niche environment in which stem cells reside has been identified as a critical component to the effectiveness of cellular engraftment and differentiation [2]. Therefore, delivering cardiac competent progenitors into a nurturing environment remains the focus of ongoing discovery pipelines.
To enable this outcome, pluripotent stem cell sources including embryonic stem cells (ESC) or their bioengineered counterparts, iPSCs, offer a significant advantage for cardiac regeneration due to their high proliferative as well as tissuespecific differentiation capacity. However, their pluripotent state carries the major risk of uncontrolled growth resulting in teratoma formation or disorganized nonfunctional tissues $[3,4]$. In order to address this issue, methods are being developed to eliminate contaminating undifferentiated cells from the therapeutic cocktails, including the use of genotoxic agents [5]. Furthermore, controlling the environment with biomaterials may offer strategic advantages to directing the safety and efficacy of pluripotent cell-based therapeutics [6]. 
Thus, identifying the extracellular components that naturally guide primitive stem cells may offer novel insight to the bioengineering requirements to further advance this field.

The environment in which progenitor cells grow is contingent upon the surrounding extracellular matrix (ECM) to provide signals, support, and a physical environment that promotes growth and differentiation. Some cardiac cell ECMs that have been studied include matrigel, a tumor isolated protein preparation, collagen, an ECM protein, fibrin, a clotting protein, synthesized polymers, and decellularized adult organ scaffolds. Herein, we aimed to determine the feasibility of applying embryonic cardiac scaffold to support pluripotent stem-cell-derived progenitors to differentiate into functional tissues. Similar approaches utilizing nonembryonic natural matrix have allowed the dissection of ECM to direct cells to become specialized cytotypes [7-10]. Adult organs that have been successfully decellularized in order to produce natural scaffolding for stem cells include kidney, lung, liver, pancreas, and heart [11-15]. Cardiac tissue has been created using adult heart support structures to sustain growth, differentiation, and tissue repopulation [16]. Adult cardiac scaffolds produced by decellularizing strategies have been perfused with cardiac progenitors giving rise to beating heart tissue $[13,16,17]$.

Here we isolate murine embryonic hearts and decellularize them via a perfusion and agitation method in order to obtain cardiac scaffolds that retained structural integrity despite the fragility of developing tissues. Once the embryonic cardiac scaffold was produced, various populations of stage-specific progenitor cells were seeded in order to determine the best developmental time point to generate cardiac tissue within the manufactured embryonic scaffold. By matching ESC-derived progenitors with a stage appropriate embryonic scaffold, this strategy may provide optimal growth and differentiation conditions to allow for a more populous growth of cardiac cells. Furthermore, this novel strategy may provide a platform to further identify stage-specific and tissue-specific signaling components to optimize nextgeneration applications for targeted cardiac regeneration.

\section{Methods}

2.1. Perfusion Decellularization of Embryonic Mouse Heart. All animal experiments were performed in accordance with US Animal Welfare Act guidelines and policies and approved by the Mayo Clinic Institutional Animal Care and Use Committee. Embryos at day 18.5 gestation (E18.5) were harvested from pregnant CD1 females (Harlan Labs). Embryos were placed in sterile PBS in a Petri dish and dissected from the uterine membrane. Embryos were sacrificed by decapitation prior to further intervention. Using an open chest procedure, the heart and ascending aorta were exposed in the embryo chest cavity. A perfusion decellularization method was used in which a suture was tied around the base of the aorta near the base of the heart, the aorta was cut halfway through vessel width proximal to the first branch and cannulated, and the suture was tied to hold the cannula in place. The heart was then removed from the chest cavity and hung on the perfusion apparatus. Perfusion occurred with $0.25 \%$ sodium dodecyl sulfate (SDS) and DNase $(0.5 \mathrm{mg} / \mathrm{mL})$ solution at room temperature for 5 hours followed by a 2-hour wash with distilled water and penicillin-streptomycin $(10 \mu \mathrm{L} / \mathrm{mL})$ at room temperature.

\subsection{Agitation Decellularization of E18.5 Mouse Heart. The} collection of E18.5 embryos was as outlined in the above procedure with the dissected heart removed from the chest cavity and placed in a $50 \mathrm{~mL}$ falcon tube. The agitation decellularization used was based on a modified protocol by Liao and collaborators [18]. Hearts were placed in the agitation solution containing $0.25 \%$ SDS and DNase $(0.5 \mathrm{mg} / \mathrm{mL})$ and incubated on a shaker at $153 \mathrm{~min}^{-1}$ for 5 hours at room temperature. Hearts underwent a 2-hour wash with distilled water and penicillin-streptomycin $(10 \mu \mathrm{L} / \mathrm{mL})$ at room temperature.

2.3. Collection of E16.5 Cardiac Cells. The collection of E16.5 embryos followed the same procedure as outlined above. Upon opening the chest, the heart was exposed and the atria were removed from the ventricular tissue and the ventricles were placed in sterile PBS in a $15 \mathrm{~mL}$ falcon tube. Ventricles were washed with PBS twice after collection and minced into small pieces prior to being transferred into a $50 \mathrm{~mL}$ falcon tube with $2 \mathrm{~mL}$ pancreatin- $(1 \mathrm{mg} / \mathrm{mL})$ collagenase $(0.5 \mathrm{mg} / \mathrm{mL})$ enzyme solution. Solution was triturated and incubated at $37^{\circ} \mathrm{C}$ for 10 minutes with intermediate agitation. Solution was then triturated with an additional $2 \mathrm{~mL}$ of enzyme solution. This process was repeated until no visible clumps remained in solution or until $10 \mathrm{~mL}$ of enzyme solution had been added. Serum containing cell culture medium was added in equal volume to the enzyme solution to inactivate the enzymes. Cell suspension was centrifuged at $1000 \mathrm{rpm}$ for 4 minutes at room temperature. The resulting cell pellet was then collected and placed in serum-containing cell culture media.

2.4. Recellularization of E18.5 Heart Scaffolds. Decellularized hearts were placed into a well in a low adherence 24-well plate containing $1 \mathrm{~mL}$ cell differentiation medium (DMEM supplemented with 20\% FBS). Approximately 300,000 cells (mESCs) were placed dropwise on top of heart in a $50 \mu \mathrm{L}$ suspension. Cells and hearts were allowed to rest for 2 minutes before being placed in incubator at $37^{\circ} \mathrm{C}$ on an oscillator at $100 \mathrm{~min}^{-1}$. Serum-containing cell culture medium was changed every second day.

\subsection{Histology and Immunofluorescence of Murine Recellular-} ized Hearts. Hearts were frozen using isopentane in OTC cryopreservation media and stored at $-80^{\circ} \mathrm{C}$ until sectioning. Hearts were sectioned to be $4 \mu \mathrm{m}$ thick and stained with hematoxylin and eosin (H\&E) and Trichrome. Four $\mu \mathrm{m}$ sections were also used for immunofluorescence. For this, sections were incubated with $1 \%$ Triton X-100 solution for 30 minutes at room temperature and blocked for 3 hours at room temperature with Superblock prior to the addition of primary antibodies. Antibodies used were $\alpha$-actinin (Sigma; 
mouse 1:500), smooth muscle actinin (SMA) (Abcam; rabbit 1:200), CD31 (Abcam; mouse 1:100), and stagespecific embryonic antibody (SSEA)-1 (Millipore; mouse 1:50). Secondary antibodies used were anti-mouse Alexa 568 (Invitrogen; 1:250) and anti-rabbit Alexa 488 (Invitrogen; $1: 250$ ). Samples were then stained with $4^{\prime}, 6$-diamidino-2phenylindole (DAPI) and fixed in 5\% paraformaldehyde (PFA) at room temperature and stored at $4^{\circ} \mathrm{C}$ until imaging.

2.6. Characterization of E16.5 Cells. Isolated cells were placed in suspension onto laminin-coated cover glasses and allowed to sit overnight at $37^{\circ} \mathrm{C}$. After a monolayer was seen on the cover glass, cells were washed with PBS, fixed with 5\% PFA for five minutes at room temperature, and stored in $\mathrm{PBS}$ at $4^{\circ} \mathrm{C}$ until immunofluorescence. Cells were incubated in $1 \%$ Triton $\mathrm{X}-100$ solution for 30 minutes at room temperature and then blocked for 3 hours with Superblock at room temperature. Primary antibodies were added and cells were stored at $4^{\circ} \mathrm{C}$ overnight. Antibodies used were $\alpha$-actinin (Sigma; mouse 1:500 dilution), Ki67 (Abcam; rabbit 1:200), connexin 43 (Millipore; rabbit 1:200), SMA (Abcam; rabbit 1:200 dilution), CD31 (Abcam; mouse 1:100), cardiac troponin (cTnT) $\operatorname{IgG}_{1}$ (Thermoscientific; mouse 1:200), and MLC2v (Proteintech; rabbit 1:200). Secondary antibodies used were anti-mouse Alexa 568 (Invitrogen; 1:250), anti-rabbit Alexa 488 (Invitrogen; 1:250), anti-rabbit 568 Alexa (Invitrogen; $1: 250$ ), and anti-mouse $\mathrm{IgG}_{1}$ Alexa 488 (Invitrogen; 1:250). Samples were stained with DAPI and fixed in 5\% PFA. Cover glasses with cells were mounted onto slides with ProLong Gold antifade reagent (Invitrogen) and stored at $4^{\circ} \mathrm{C}$ until imaging.

2.7. Characterization of Differentiated R1 ESC. Spontaneous differentiation of mouse (m)ESCs was performed using an embryoid body (EB) system. At passage 8, mESCs were trypsinized ( $0.25 \%$ trypsin, $5 \mathrm{~min})$ and resuspended in differentiation medium. EBs containing 2000 cells were generated via centrifugation of mESCs into an Aggrewell plate (STEMCELL Technologies) according to manufacturer's instructions. In brief, mESCs were spun down into the Aggrewells at day 0. Following 24 hours, the media was replaced and at 48 hours, EBs were flushed from the wells and allowed to float in culture for the next 48 hours. At day 5, EBs were plated at $\sim 30 \mathrm{EBs}$ per $100 \mathrm{~mm}$ gelatincoated culture dish, in triplicate. $25 \mathrm{EBs}$ were split into five groups of $5 \mathrm{EBs}$ and fixed in 5\% PFA. EBs were stored in PBS at $4^{\circ} \mathrm{C}$ until immunofluorescence. The remaining EBs were digested with Collagenase B ( $1 \mathrm{mg} / \mathrm{mL}$, Roche) enzyme solution at $37^{\circ} \mathrm{C}$. EBs were transferred into $50 \mathrm{~mL}$ falcon with $200 \mu \mathrm{L}$ of enzyme solution, triturated, and placed in water bath at $37^{\circ} \mathrm{C}$ for 5 minutes. $200 \mu \mathrm{L}$ additional enzyme solution was added after five minutes and EBs were triturated and returned to water bath. This process was repeated until no visible clumps remained in solution. Cell medium was added in equal volume to the enzyme solution; cells were collected after centrifugation at $1000 \mathrm{rpm}$ for 4 minutes and resuspended in $250 \mu \mathrm{L}$ of differentiation medium. Cells were placed in $50 \mu \mathrm{L}$ drop suspension onto gelatin-coated cover glass and allowed to sit at $37^{\circ} \mathrm{C}$ for 1 hour at which time $1 \mathrm{~mL}$ of media was added to the wells. After overnight incubation at $37^{\circ} \mathrm{C}$, cells were washed with PBS and fixed in 5\% PFA. Cells were stored at $4^{\circ} \mathrm{C}$ in $\mathrm{PBS}$ until immunofluorescence.

Immunofluorescence was done on fixed floating EBs as well as digested EBs fixed as cells. Samples were incubated in $1 \%$ Triton X-100 solution for 30 minutes at room temperature and then blocked for 3 hours with Superblock at room temperature. Primary antibodies were added and cells were stored at $4^{\circ} \mathrm{C}$ overnight. Antibodies used were $\alpha$-actinin (Sigma; mouse 1:500), Ki67 (Abcam; rabbit 1:200), connexin 43 (Millipore; rabbit 1:200), SMA (Abcam; rabbit 1:200),

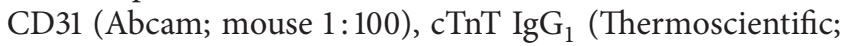
mouse 1:200), myosin light chain 2-ventricular (MLC2v, Proteintech; rabbit 1:200), and SSEA1 (Millipore; mouse 1:50). Secondary antibodies used were anti-mouse Alexa 568 (Invitrogen; 1:250), anti-rabbit Alexa 488 (Invitrogen; $1: 250$ ), anti-rabbit Alexa 568 (Invitrogen; 1:250), and antimouse $\mathrm{IgG}_{1}$ Alexa 488 (Invitrogen; 1:250). Samples were stained with DAPI and fixed in 5\% PFA. Cover glasses were mounted onto slides with ProLong Gold antifade reagent (Invitrogen) and floating EBs were left in Eppendorf with PBS. All samples were stored at $4^{\circ} \mathrm{C}$ until imaging.

2.8. Electron Microscopy. Hearts were placed in Trumps solution for 3 weeks at $4^{\circ} \mathrm{C}$. The electron microscopy followed established protocols [19].

\section{Results}

3.1. Decellularization of Embryonic Hearts Produces Intact Cardiac Scaffolds. After harvesting E18.5 embryonic hearts, two decellularization methods were compared: $5 \mathrm{hr}$ perfusion with $0.25 \%$ SDS and DNase $(0.5 \mathrm{mg} / \mathrm{mL})$ or $5 \mathrm{hr}$ agitation with $0.25 \%$ SDS and DNase $(0.5 \mathrm{mg} / \mathrm{mL})$. Both methods produced intact cardiac scaffolds with no remaining cellular remnants as confirmed by H\&E staining and immunofluorescence with SMA, endothelial (CD31), actinin, and DAPI as shown in Figure 1. The agitation method of decellularization did not damage the integrity of the scaffold and preserved heart structures, whereas the perfusion method did not reliably preserve structural integrity of the scaffold (see Supplemental A in Supplementary Material available online at http://dx.doi.org/10.1155/2014/625164). Moreover, perfusion-derived scaffolds were extremely fragile and minor manipulations resulted in tears, including the challenging operation of cannula removal. Due to the quality of the matrix and better preservation of the cardiac structure, the agitation method proved to be a simple protocol that reliably produced a practical scaffold for subsequent experiments.

\subsection{Higher SDS Concentration Results in Increased Damage} of the Cardiac Scaffolds. In order to optimize the agitation protocol, E18.5 hearts were decellularized with varying concentrations of SDS ranging from $0.25 \%$ to $1 \%$. All SDS concentrations were successful at removing cellular remnants. However, the scaffolds produced with high levels of SDS became difficult to handle due to increased adherence 


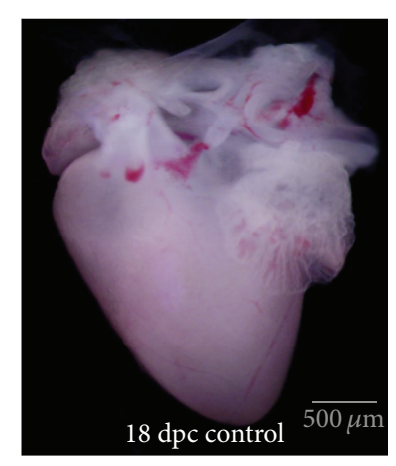

(a)

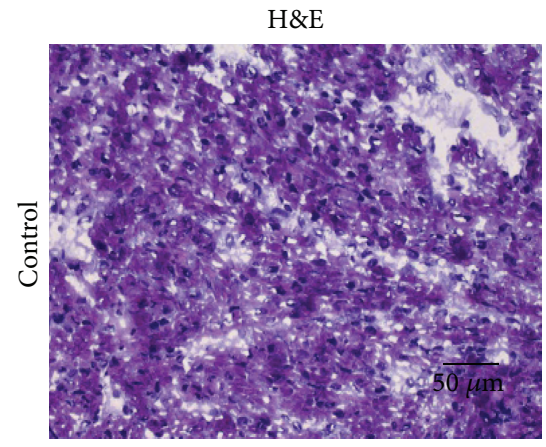

SMA/DAPI
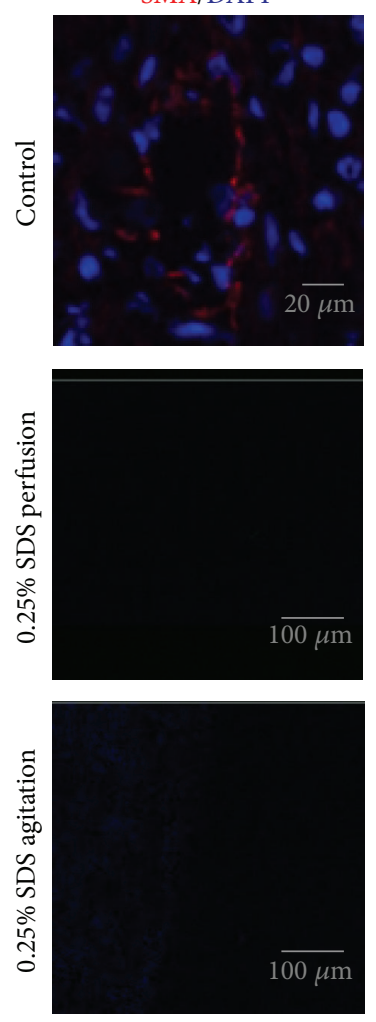

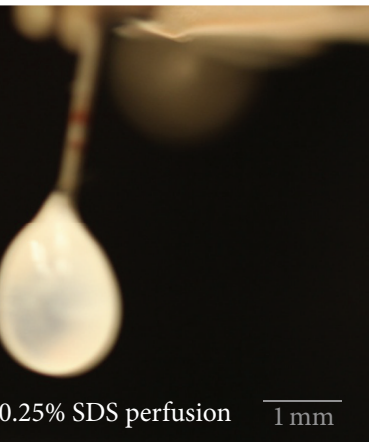

(b)

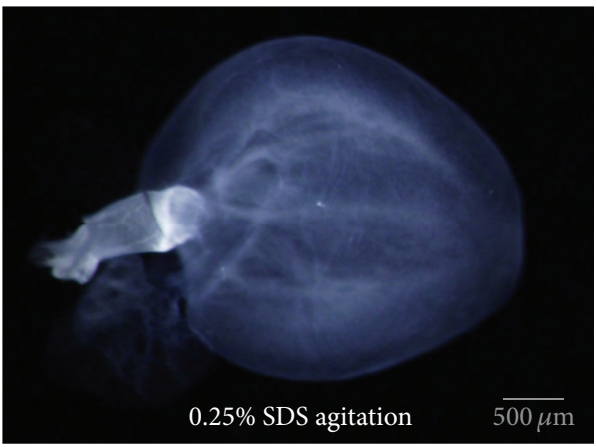

(c)
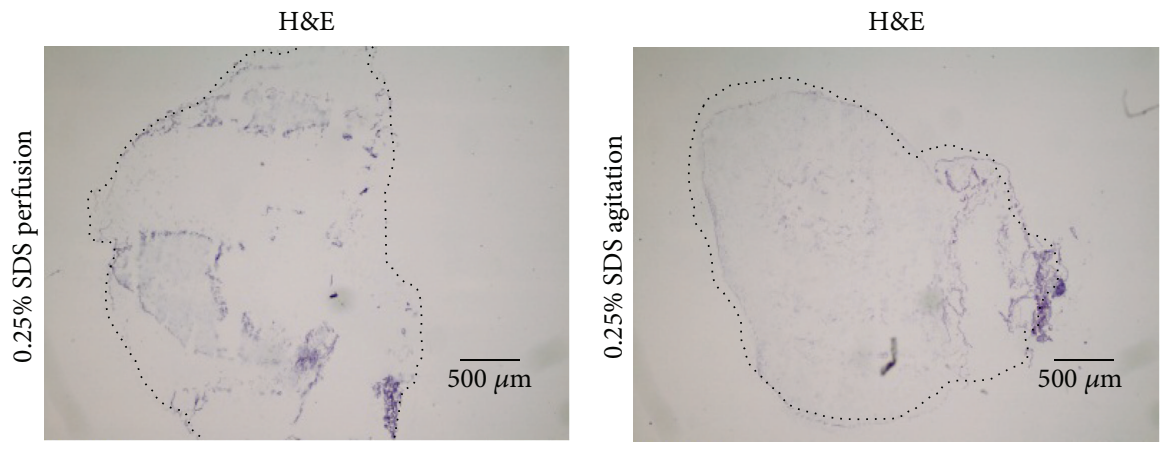

(d)
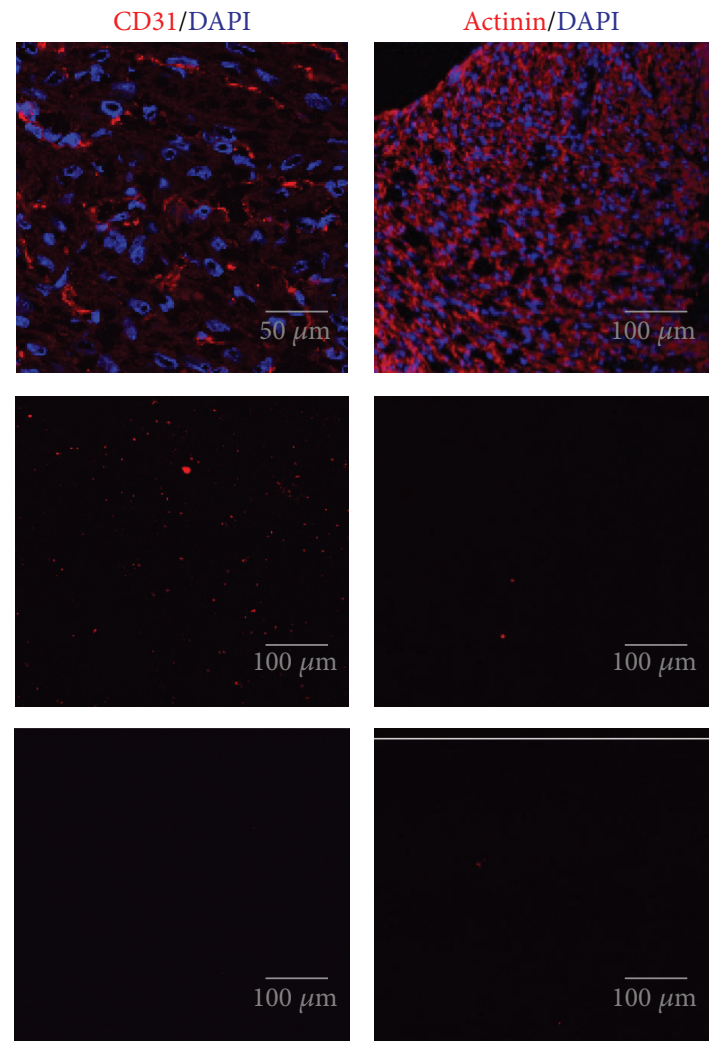

(e)

Figure 1 

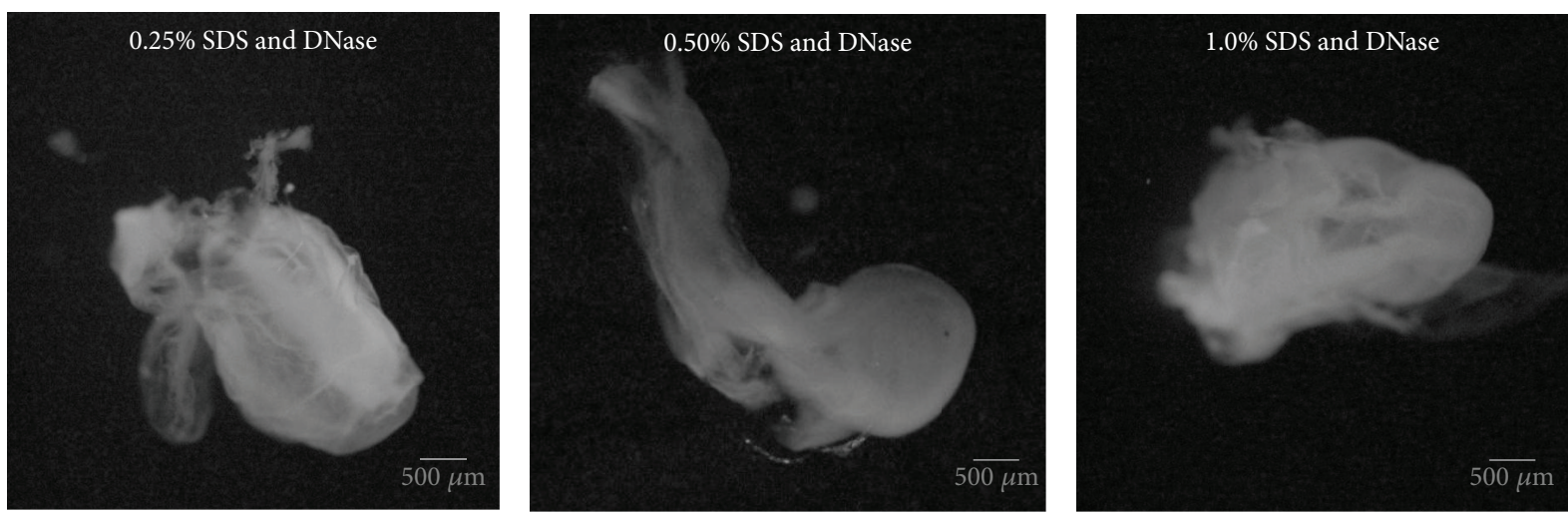

(a)
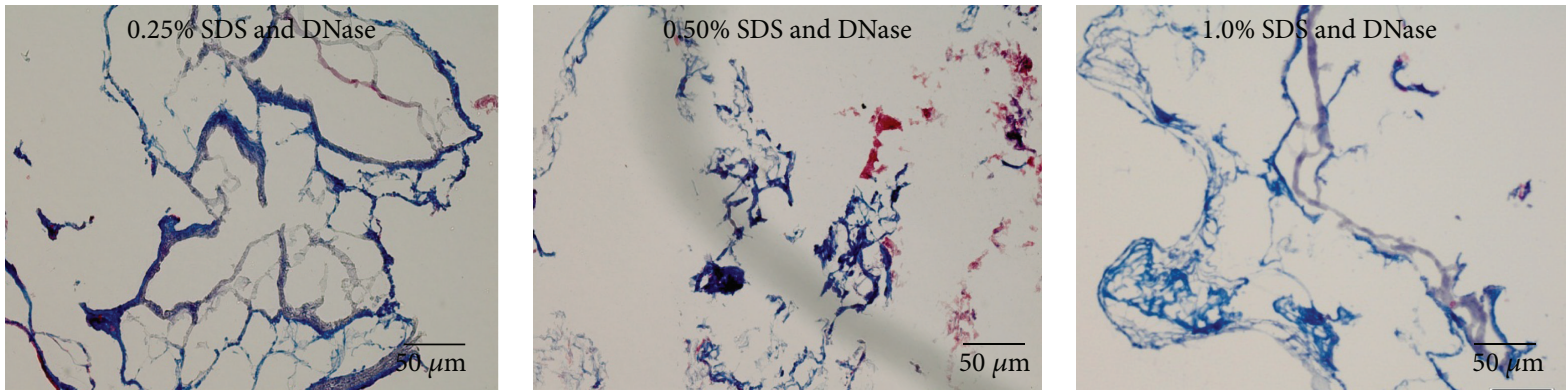

(b) to dissection tools and dishes. Additionally, these scaffolds tended to fold thus losing preserved cardiac structures. As seen in Figure 2, Masson's Trichrome staining revealed loss of matrix integrity with increasing concentrations of SDS. Cardiac structure was retained with $0.25 \%$ SDS and collagen fibers maintained long filamentous networks, as compared to the $0.5 \%$ and $1 \%$ SDS in which the cardiac structure was lost due to scaffold folding and short broken collagen filaments. Therefore, agitation of E18.5 hearts in $0.25 \%$ SDS is sufficient to remove cellular remains while still preserving cardiac ECM macro- and microstructure.

\subsection{Recellularization of Cardiac Scaffolds with E16.5 Ventric-} ular Cells Reconstitutes Beating Cardiac Tissue. Embryonic hearts were used to collect viable single cells that could be used as positive control for recellularization protocols. We first analyzed cells isolated from E16.5 ventricles with immunofluorescence of connexin 43, actinin, MLC2 $v$, and troponin expression (Figure 3). The endothelial marker CD31 was expressed in $27 \%$ of cells while smooth muscle marker SMA was expressed in $25 \%$ of cells. Of the isolated E16.5 ventricular cells characterized, $18 \%$ were cardiomyocytes according to cardiac-specific markers. Actively dividing cells were identified using Ki67 staining and revealed $43 \%$ of cells were dividing; of these dividing cells 14\% were cardiomyocytes (Supplemental B). E16.5 ventricular cells were successfully reseeded into embryonic cardiac scaffolds as shown in Figure 3 by immunofluorescence with SMA, actinin, CD31, and DAPI. The actinin within cardiomyocytes present in the reseeded cardiac scaffolds showed cardiac striations. Moreover, areas of spontaneous beating corresponding to recellularized cardiac tissue were produced within the bioengineered construct $20 \mathrm{hrs}$ after the addition of E16.5 cells into the scaffolds (Supplemental C). Macroscopic appearance of the supporting matrix evolved from translucent to opaque following cell inclusion into the construct. This was further documented by H\&E and toluidine blue staining of the reseeded scaffolds revealing a highly cellular environment as well as a dense tissue newly formed onto the host matrix. Collagen structures became more physically compact after incubation of the scaffold with E16.5 cells, as shown in electron microscopy (EM). EM micrographs revealed presence of sarcomeres and abundant mitochondria in the cardiomyocytes within the recellularized construct. Mature organelles such as Golgi apparatus and endoplasmic reticulum were clearly identified in the cells repopulating the decellularized scaffold as shown in Figure 4. Therefore, embryonic decellularized cardiac constructs create 


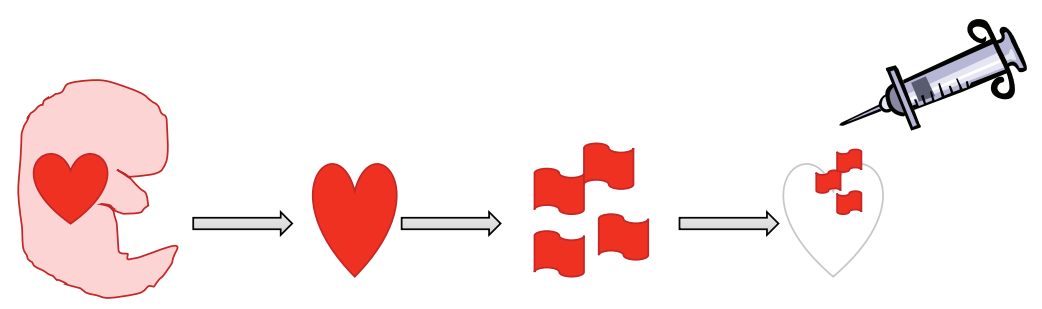

(a)
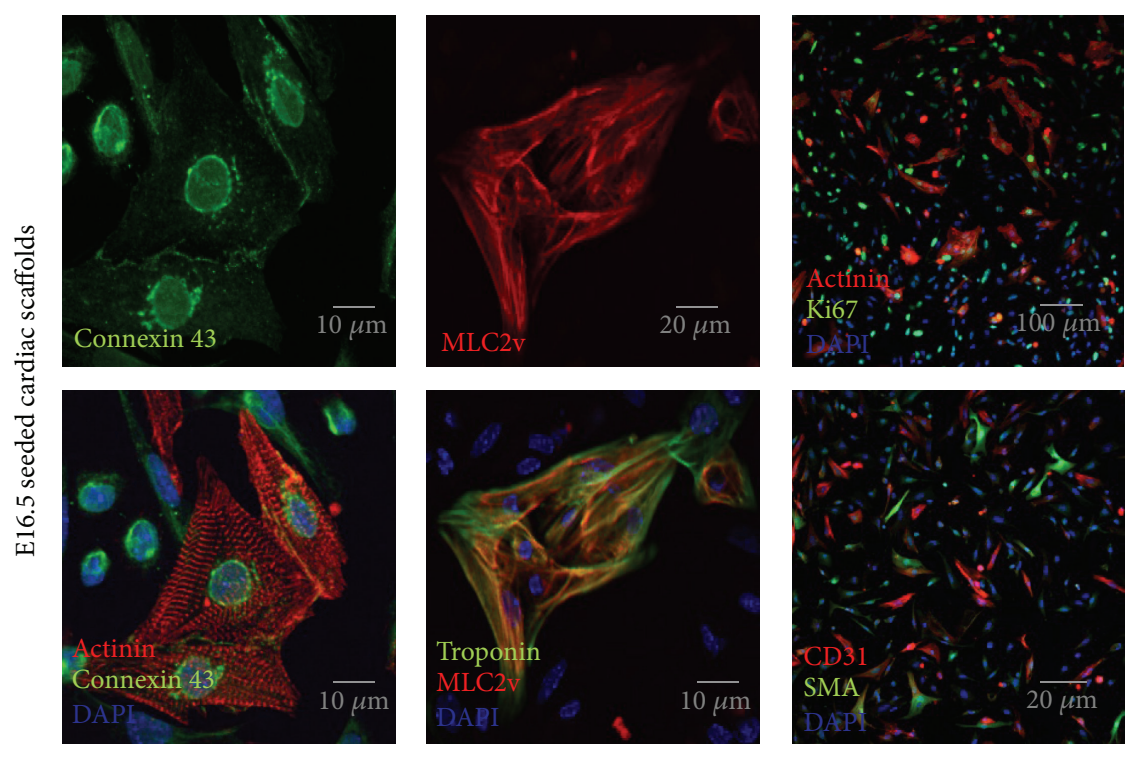

(b)
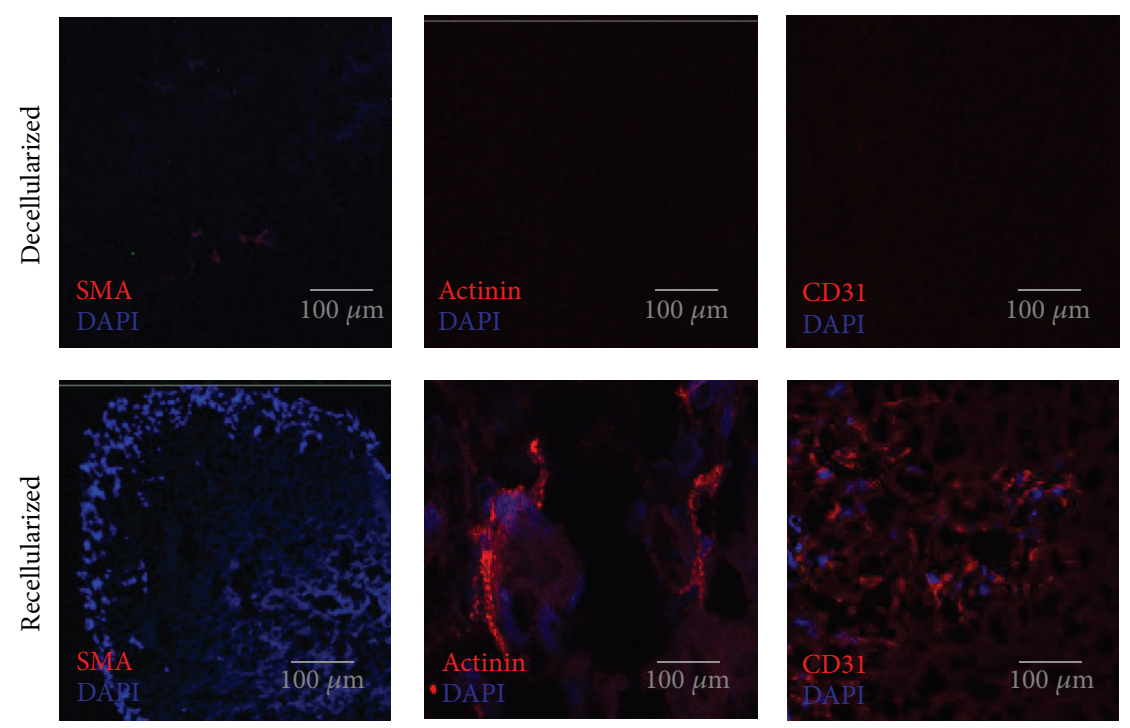

(c)

FIGURE 3

a propitious microenvironment that supports migration and integration of immature cardiac cytotypes.

3.4. Cardiac Scaffolds Provide a Host Environment for Pluripotent Stem-Cell-Derived Progenitors to Produce Beating Cardiac Tissue. Following optimization of the recellularization protocols using age-matched single cells, we focused on application of pluripotent stem-cell-derived progenitors as the cell source to bioengineer beating cardiac tissue. Engraftment and differentiation of undifferentiated pluripotent stem cells (day 0 ) as well as progenitors after spontaneous EB-based differentiation for 5 and 9 days were longitudinally compared using immunofluorescence of the reseeded cardiac scaffolds as 

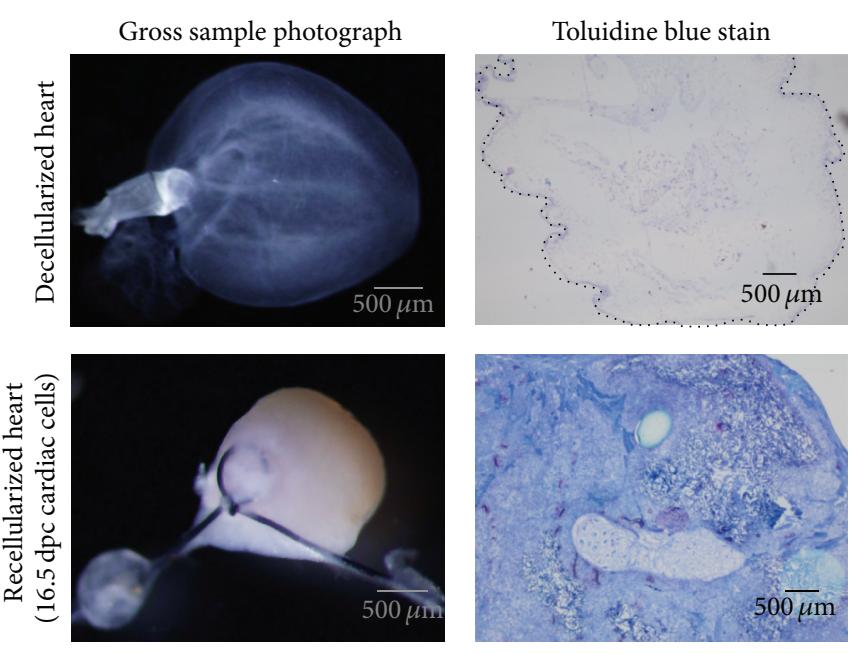

(a)
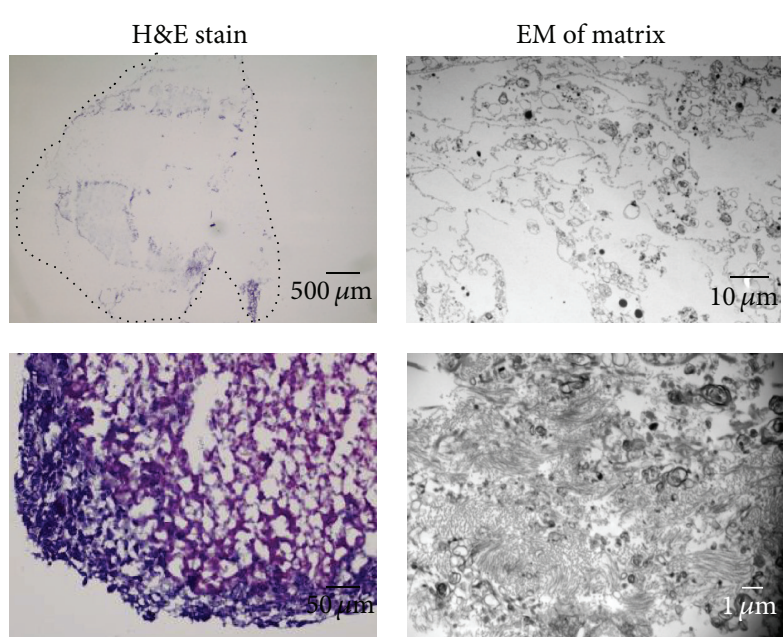

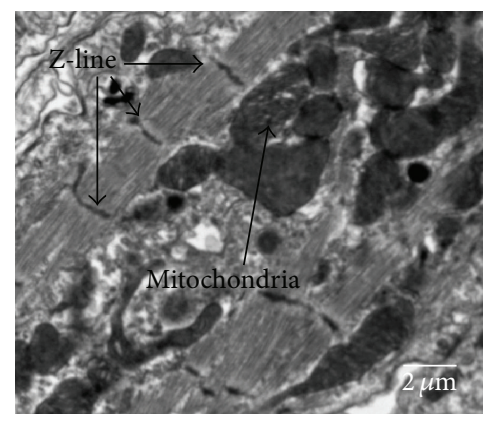

(b)

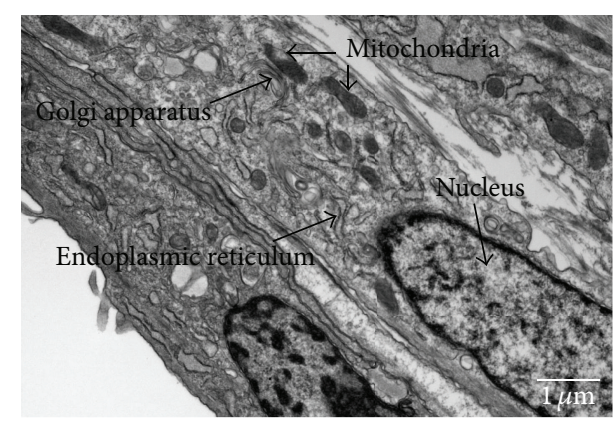

(c)

Figure 4

shown in Figure 5. Scaffolds recellularized with day 0 mESCs showed high amounts of cells with SSEA1, low levels of actinin, scant cellularization, and no CD31 or SMA. In parallel, cardiac scaffolds reseeded with day 9 progenitors showed low amounts of SSEA1 and cellularization and revealed no actinin, CD31, or SMA. Conversely, cardiac scaffolds reseeded with day 5 progenitors derived from mESCs revealed small amounts of SSEA1, high levels of cellularization, and low amounts of actinin after one week. Long-term incubation of embryonic cardiac scaffolds with day 5 progenitors for 24 days resulted in spontaneously beating constructs. These samples had low amounts of SSEA1, SMA, and CD31 and had high amounts of cellularization and actinin. Furthermore, H\&E and phosphotungstic acid haematoxylin (PTAH) staining of these bioengineered constructs revealed a densely populated matrix with abundance of cells as seen in Figure 5. Therefore, cardiac progenitors derived from pluripotent stem cells through spontaneous differentiation are sufficient to produce beating cardiac constructs when incubated together with embryonic cardiac scaffolds.

\section{Discussion}

Cardiac regenerative medicine aims to repair damaged heart muscle through the use of stem cells, yet producing functional tissues remains a challenge. Using a cadre of bioengineering approaches, there are multiple emerging strategies in which cell-based repair processes can be applied. Many of the novel approaches are leveraging the niche microenvironment with stem cells, as the functional tissue is a direct product of the interaction between these essential components. The use of a decellularized cardiac scaffold to drive progenitor cells toward cardiac phenotypes is among the most alluring concepts for transformative applications in cardiac transplant medicine and surgery. Pioneering research with adult cardiac scaffolds has provided proof-of-principle studies that demonstrate the feasibility of this approach. The present study focused on matching the developmental stages of a cardiac scaffold with stage-appropriate progenitor cells in order to determine the impact of scaffold-cell matching on the bioengineering process of cardiac tissue. By pairing the embryonic scaffold with pluripotent stem-cell-derived progenitor cells, the environment can optimize tissue-specific differentiation and functional applications for discovery sciences and translational platforms.

Pluripotent embryonic stem cells provide optimal growth and differentiation for bioengineering tissue-specific lineages as they are naturally designed to accommodate the developmental requirements during de novo tissue formation. By utilizing an embryonic heart matrix paired with primitive progenitor cells derived from embryonic stem cells, we aimed to enhance the guided growth of functional cardiac tissue. 


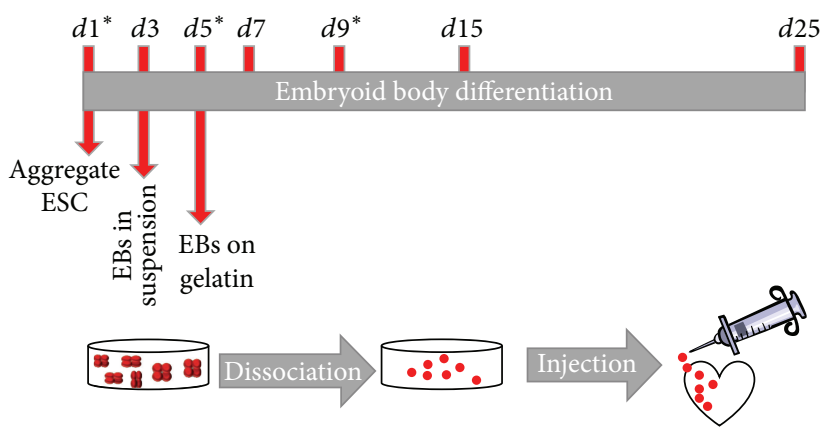

(a)
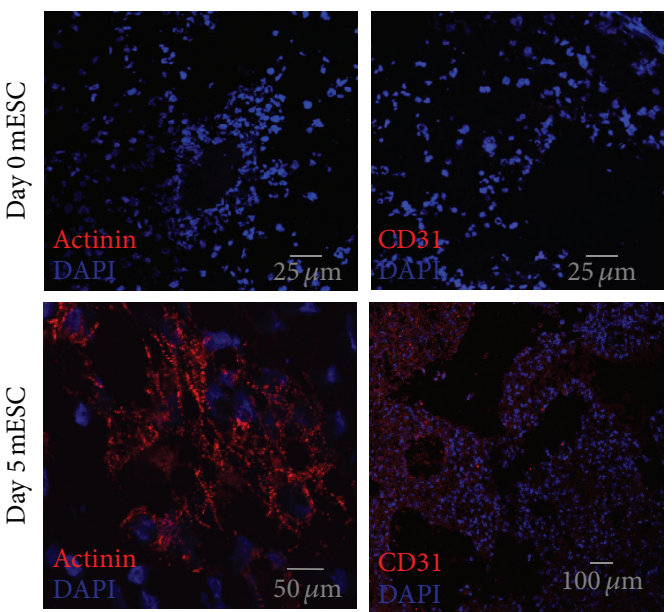

\begin{tabular}{|l|c|c|c|c|c|c|}
\hline $\begin{array}{l}\text { mESC sample } \\
\text { (days cells } \\
\text { spent with } \\
\text { heart) }\end{array}$ & Actinin & CD31 & SMA & SSEA1 & Cellularization & $\begin{array}{c}\text { Beating } \\
\text { activity }\end{array}$ \\
\hline $\begin{array}{l}\text { Day 0 }(11 \\
\text { days) }\end{array}$ & ++ & 0 & 0 & ++++ & ++ & No \\
\hline Day 5 (7 days) & + & 0 & 0 & ++ & ++ & No \\
\hline $\begin{array}{l}\text { Day 5 (24 } \\
\text { days) }\end{array}$ & +++ & + & + & + & ++++ & Yes \\
\hline Day 9 (7 days) & 0 & 0 & 0 & + & + & No \\
\hline
\end{tabular}

$\begin{array}{llll}\text { Amount of staining: } & & \\ 0 & \text { Not present } & +++ & \text { Good } \\ + & \text { Minimal } & ++++ & \text { Most } \\ ++ & \text { Fair } & & \end{array}$

The unmet challenge of this approach was the production of robust scaffolds from embryonic hearts that were decellularized yet maintained structural integrity. Specifically, the method to produce cardiac scaffolds was optimized to preserve cardiac structures that could provide the framework for subsequent differentiation. Multiple ages of hearts were tested including 16dpc and neonatal 4-day and 7-day hearts and it was noted that E18.5 hearts provided the optimal structural properties with a straightforward protocol. By isolating stage-specific scaffolds, we were then able to start matching (c)

(d)

FIGURE 5
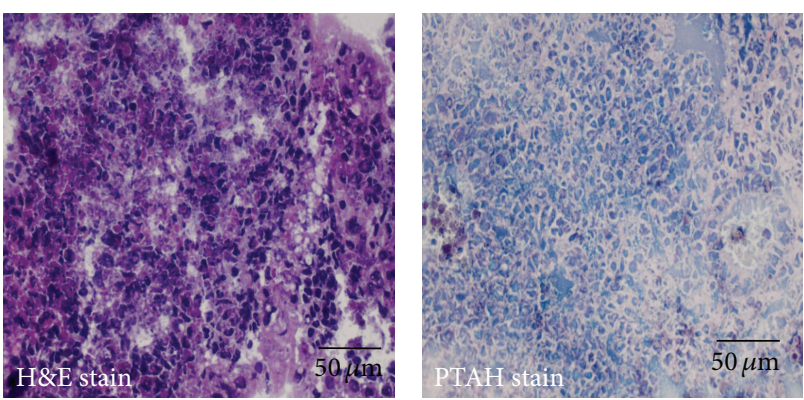

(b)
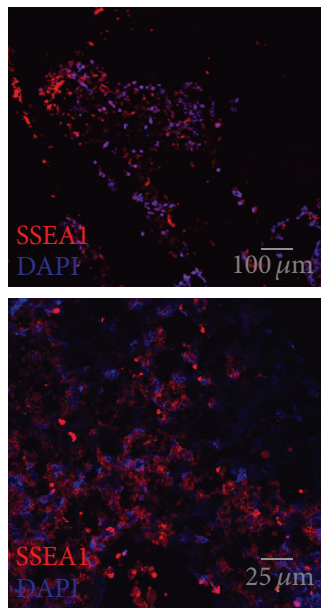

DAPL $\quad \therefore 25 \bar{m}$

stage-appropriate progenitor cells with the scaffold to determine ideal cardiac tissue formation.

Reseeding of the isolated embryonic cardiac scaffold resulted in cellular engraftment with various types of cells; however, not all cells tested produced functional maturation manifested as spontaneous beating activity. Matching of the cell type and developmental stage of the scaffold proved to be the critical point in recellularization to enable production of beating cardiac tissue. Multipotent progenitors derived after 5 days of spontaneous differentiation proved to be the 
optimal population in terms of engraftment and cardiac differentiation when combined with E18.5 cardiac scaffolds. These progenitor cells were able to engraft into the scaffold and produced beating cardiac tissue within 20 days of in situ development. It is possible that the later time point of cells was past a critical signal point and exiting the cell cycle thus proving to be not ideal for growth and guided differentiation. Cell population contained in earlier differentiation time points remained in the pluripotent state with persistent expression of SSEA1 and unresponsive to the embryonic cardiac scaffold. Thus, matching the ideal developmental stages of both the host environment and the progenitor cells proves to be an important component to successful differentiation of functional cardiac tissues.

An inherent challenge to this approach is that embryonic stage tissues are small and impractical for surgical applications as well as being limited to xenografts as human constructs would not be feasible. Therefore, this current strategy is ideally suited for discovery sciences to better understand the essential components of the matrix required to support developmental maturation. Furthermore, it could be envisioned that miniature cardiac tissue constructs could be applied in the setting of pharmaceutical safety testing to model the tissue requirements beyond the limitations of cardiomyocytes in monolayer assays. Further comparison with adult constructs and bioengineered materials will accelerate the discovery of embryonic components that may be extracted and applied to clinical-grade applications aiming towards therapeutic endpoints.

Overall this research accomplished three important milestones that can be applied towards cardiac regeneration research: production of an embryonic scaffold, optimized methods to preserve this scaffold, and recellularization protocols to produce functional beating cardiac tissue. These milestones have led to the advance concept that scaffoldcell matching is critical in the differentiation and maturation of bioengineered cardiac tissues. By optimizing the developmental stage of scaffold or the niche environment with the progenitor cells required for reseeding the natural environment during cardiac development can be better recapitulated. Therefore, the biologically inspired engineering process can be optimized to enhance the overall population of functional cardiac tissues and provide a platform for subsequent cardiac regenerative studies.

\section{Conflict of Interests}

The authors declare that there is no conflict of interests regarding the publication of this paper.

\section{Acknowledgments}

The authors would like to thank Lois Rowe for her histology expertise, Jon Nesbitt for his animal expertise, and the Electron Microscopy Core Facility for their expertise.

\section{References}

[1] B. C. du Pre, P. A. Doevendans, and L. W. van Laake, "Stem cells for cardiac repair an introduction," Journal of Geriatric Cardiology, vol. 10, pp. 186-197, 2013.

[2] A. Alcon, E. Cagavi Bozkulak, and Y. Qyang, "Regenerating functional heart tissue for myocardial repair," Cellular and Molecular Life Sciences, pp. 1-22, 2012.

[3] J. Leor, S. Gerecht, S. Cohen et al., "Human embryonic stem cell transplantation to repair the infarcted myocardium," Heart, vol. 93, no. 10, pp. 1278-1284, 2007.

[4] T. A. Prokhorova, L. M. Harkness, U. Frandsen et al., "Teratoma formation by human embryonic stem cells is site dependent and enhanced by the presence of Matrigel," Stem Cells and Development, vol. 18, no. 1, pp. 47-54, 2009.

[5] A. Smith, N. G. Nelson, S. Oommen et al., "Apoptotic susceptibility to DNA damage of pluripotent stem cells facilitates pharmacologic purging of teratoma risk," Stem Cells Translational Medicine, vol. 1, pp. 709-718, 2012.

[6] V. F. M. Segers and R. T. Lee, "Biomaterials to enhance stem cell function in the heart," Circulation Research, vol. 109, no. 8, pp. 910-922, 2011.

[7] J. Cortiella, J. Niles, A. Cantu et al., "Influence of acellular natural lung matrix on murine embryonic stem cell differentiation and tissue formation," Tissue Engineering A, vol. 16, no. 8, pp. 2565-2580, 2010.

[8] P. M. Crapo, T. W. Gilbert, and S. F. Badylak, "An overview of tissue and whole organ decellularization processes," Biomaterials, vol. 32, no. 12, pp. 3233-3243, 2011.

[9] S. L. J. Ng, K. Narayanan, S. Gao, and A. C. A. Wan, "Lineage restricted progenitors for the repopulation of decellularized heart," Biomaterials, vol. 32, no. 30, pp. 7571-7580, 2011.

[10] M. Prideaux, N. Loveridge, A. A. Pitsillides, and C. Farquharson, "Extracellular matrix mineralization promotes E11/gp38 glycoprotein expression and drives osteocytic differentiation," PLoS ONE, vol. 7, no. 5, Article ID e36786, 2012.

[11] S. K. Goh, S. Bertera, P. Olsen et al., "Perfusion-decellularized pancreas as a natural 3D scaffold for pancreatic tissue and whole organ engineering," Biomaterials, vol. 34, pp. 6760-6772, 2013.

[12] H. C. Ott, B. Clippinger, C. Conrad et al., "Regeneration and orthotopic transplantation of a bioartificial lung," Nature Medicine, vol. 16, no. 8, pp. 927-933, 2010.

[13] H. C. Ott, T. S. Matthiesen, S.-K. Goh et al., "Perfusiondecellularized matrix: using nature's platform to engineer a bioartificial heart," Nature Medicine, vol. 14, no. 2, pp. 213-221, 2008.

[14] E. A. Ross, M. J. Williams, T. Hamazaki et al., "Embryonic stem cells proliferate and differentiate when seeded into kidney scaffolds," Journal of the American Society of Nephrology, vol. 20, no. 11, pp. 2338-2347, 2009.

[15] B. E. Uygun, A. Soto-Gutierrez, H. Yagi et al., "Organ reengineering through development of a transplantable recellularized liver graft using decellularized liver matrix," Nature Medicine, vol. 16, no. 7, pp. 814-820, 2010.

[16] T. Eschenhagen and W. H. Zimmermann, "Engineering myocardial tissue," Circulation Research, vol. 97, no. 12, pp. 12201231,2005

[17] J. M. Wainwright, C. A. Czajka, U. B. Patel et al., "Preparation of cardiac extracellular matrix from an intact porcine heart," Tissue Engineering C, vol. 16, no. 3, pp. 525-532, 2010. 
[18] J. Liao, J. Erinn, and M. Sacks, "Effects of decellularization on the mechanical and structural properties of the porcine aortic valve leaflet," Biomaterials, vol. 29, pp. 1065-1074, 2008.

[19] A. Martinez-Fernandez, T. J. Nelson, S. Yamada et al., "iPS programmed without c-myc yield proficient cardiogenesis for functional heart chimerism," Circulation Research, vol. 105, pp. 648-656, 2009. 


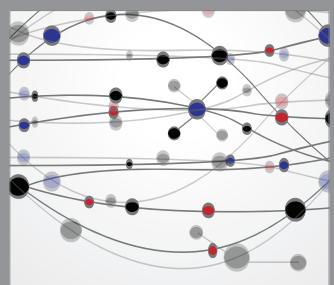

The Scientific World Journal
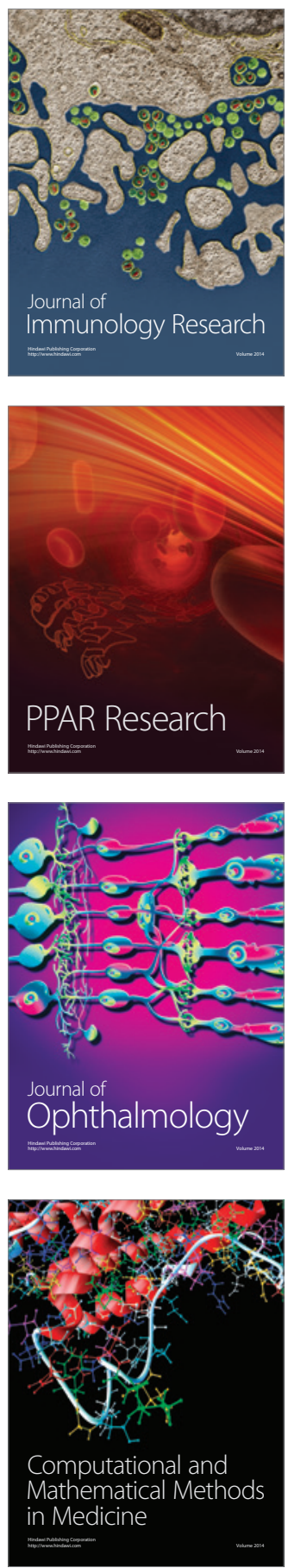

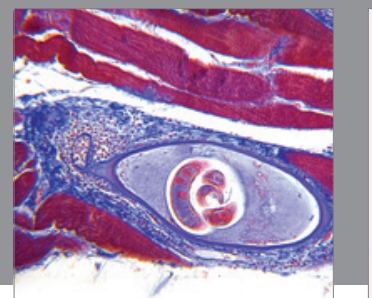

Gastroenterology

Research and Practice
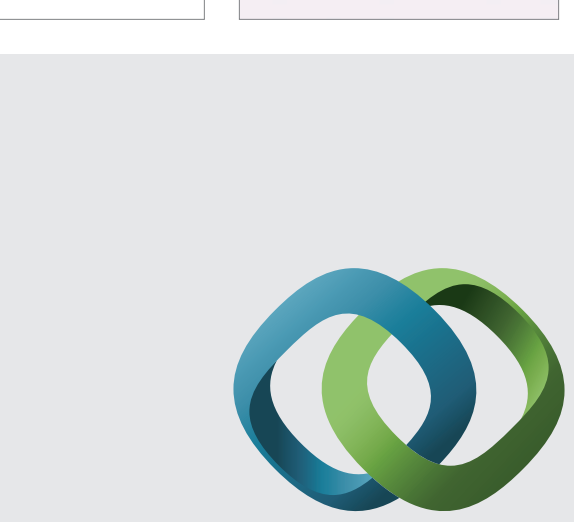

\section{Hindawi}

Submit your manuscripts at

http://www.hindawi.com
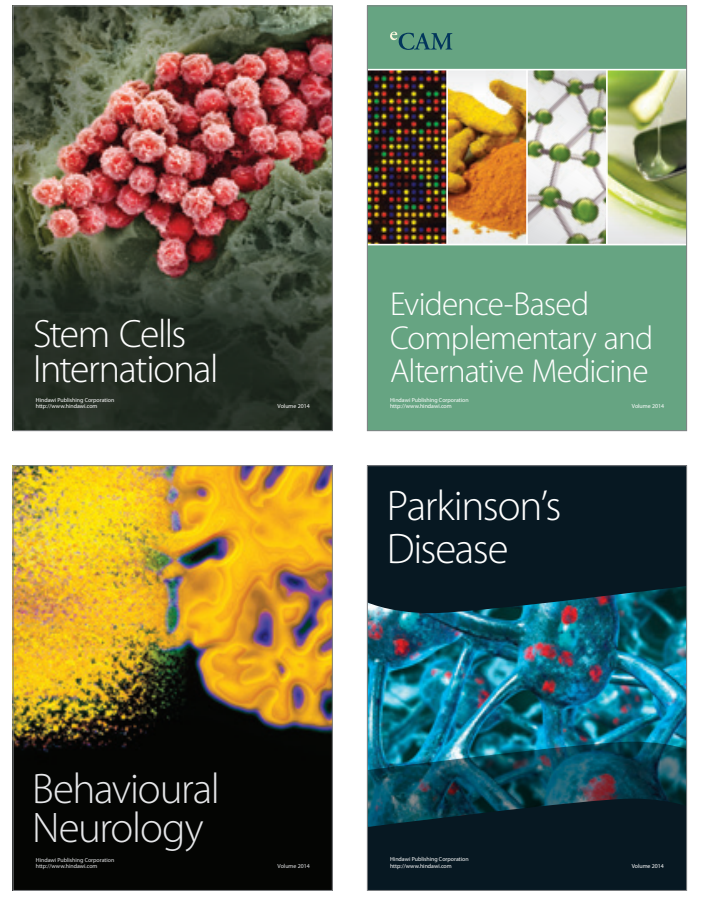
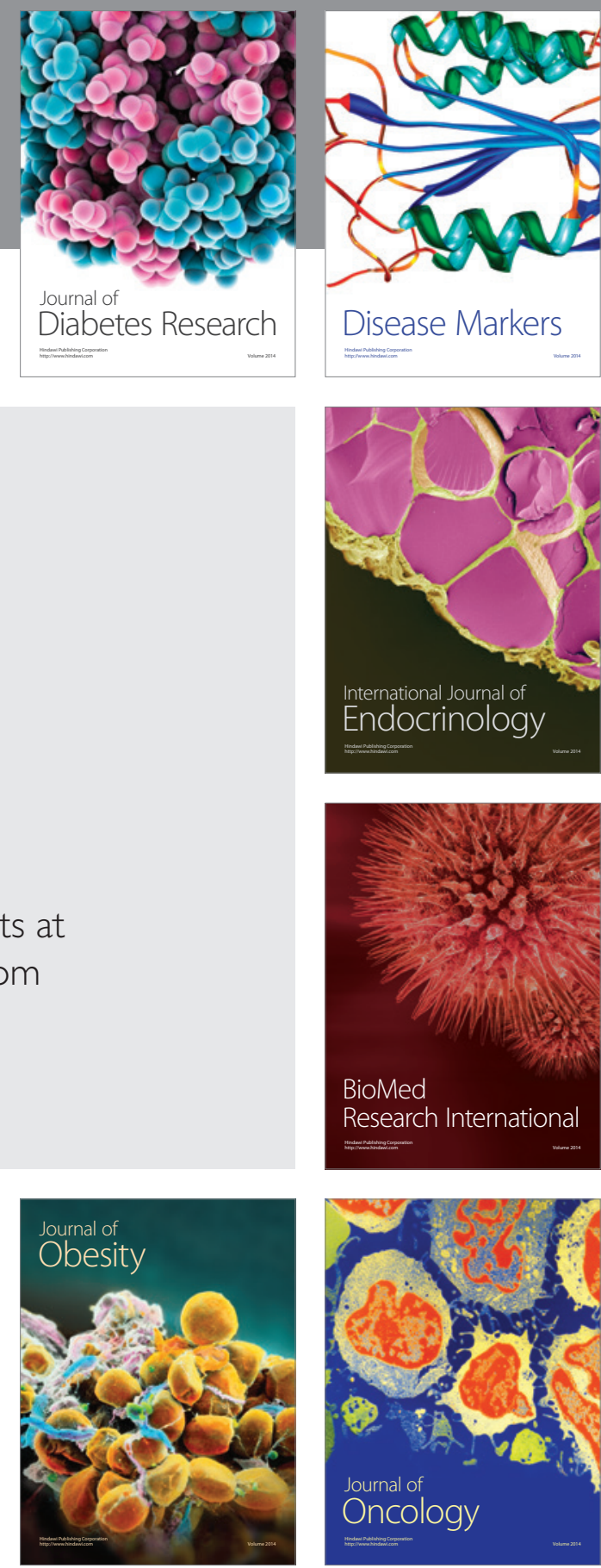

Disease Markers
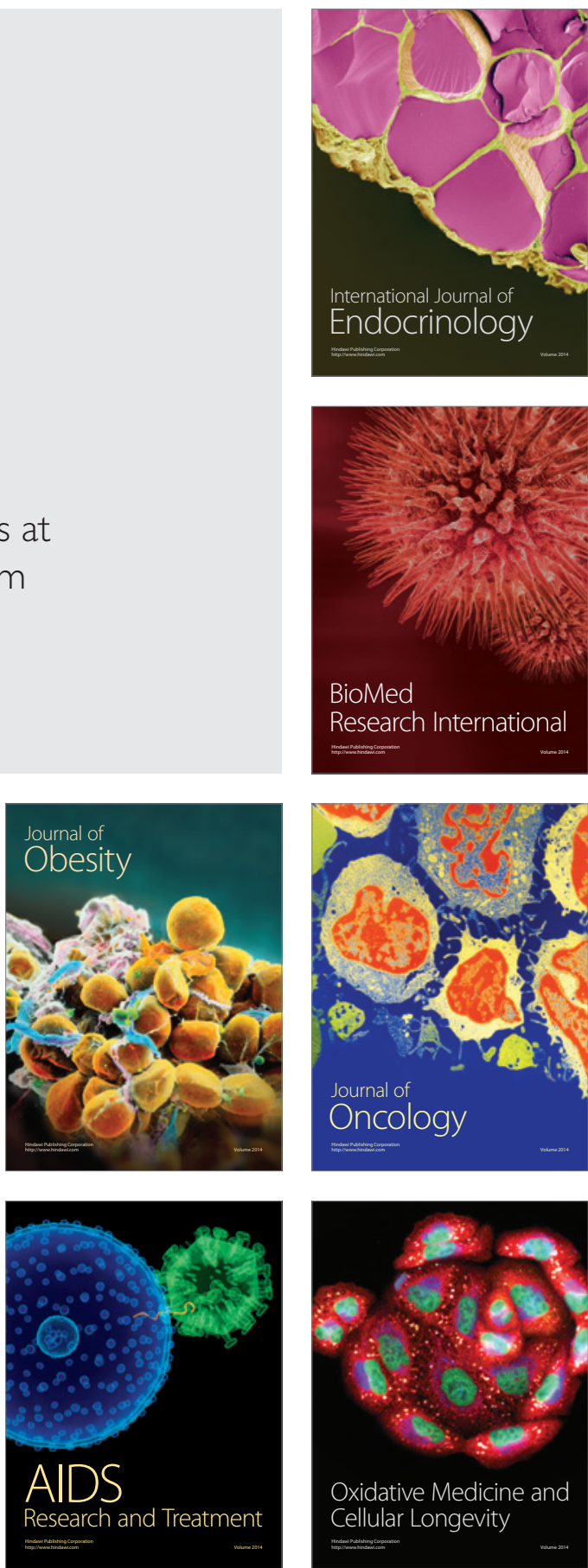